\title{
Índice de anisocitose eritrocitária (RDW): diferenciação das anemias microcíticas e hipocrômicas
}

\section{Red blood cell distribution width (RDW): differentiation of microcytic and hypochromic anemias}

\author{
Januária F. Matos ${ }^{l}$ \\ Luci M. S. Dussel \\ Rachel V. B. Stubbert ${ }^{2}$ \\ Geralda F. G. Lages ${ }^{1}$ \\ Maria das Graças Carvalho
}

\begin{abstract}
A anemia ferropriva, talassemia menor e anemia de doença crônica são as anemias microcíticas e hipocrômicas mais comuns em nosso meio. O diagnóstico diferencial das referidas anemias é de grande importância clínica; contudo, muitas vezes é complexo em virtude de concomitância de doenças, além de demandar tempo e apresentar custos significativos. Com o propósito de conferir maior simplicidade e eficiência ao diagnóstico diferencial destas anemias, o uso de índices derivados de modernos contadores automáticos tem sido sugerido. Entre estes, pode ser citado o indice de anisocitose eritrocitária $(R D W)$, que indica o grau de variabilidade do tamanho das hemácias. Neste estudo, o poder de discriminação deste índice quanto ao tipo de anemia microcítica e hipocrômica foi avaliado em um grupo de 159 pacientes sabidamente portadores de um quadro de anemia causado por deficiencia de ferro, beta talassemia menor ou uma anemia de doença crônica. Não foi observada diferença significativa para o RDW entre os três grupos de anemias microcíticas, indicando não ser este indice uma ferramenta útil para a diferenciação entre anemia ferropriva, beta talassemia menor e anemia de doença crônica. Rev. bras. hematol. hemoter. 2008; 30(2):120-123.
\end{abstract}

Palavras-chave: Anemia ferropriva; anemia de doença crônica; talassemia menor.

\section{Introdução}

As anemias microcíticas e hipocrômicas mais comuns em nosso meio são a anemia ferropriva, anemia de doença crônica e talassemia menor. ${ }^{1}$ As talassemias são anemias hereditárias causadas por uma deficiência parcial ou completa da síntese de cadeias globínicas e constituem os distúrbios genéticos mais comuns e simples no mundo inteiro, causando um grande problema de saúde pública. Uma estimativa disponível indica que 250 milhões, correspondentes a 4,5\% da população mundial, são heterozigotos para um defeito no gen da globina. ${ }^{2,3}$ Além da alteração na síntese de cadeias globínicas, os distúrbios no metabolismo do ferro também são causas de anemia microcítica e hipocrômica, sendo, neste caso, a anemia ferropriva e anemia de doença crônica aquelas mais freqüentes. A deficiência de ferro é a mais comum das alterações nutricionais na infância, não apenas nas nações em desenvolvimento, mas também em países altamente industrializados. ${ }^{4}$ Os números são assustadores, uma vez que 2 bilhões de pessoas (em torno de $30 \%$ da população mundial) são anêmicos, principalmente devido à deficiência do ferro, situação freqüentemente exacerbada pela malária e doenças parasitárias em países em desenvolvimento. ${ }^{5}$ Além disso, a deficiência do ferro possui importante impacto funcional em humanos por levar a alterações na resposta imunológica, incluindo a diminuição da atividade bactericida dos neutrófilos e do número de linfócitos, alteração no metabolismo de hormônios tireoidianos, alteração na função do músculo liso e estriado, alteração no comportamento e, também, conseqüências econômicas, tais como menor habilidade cognitiva

${ }^{1}$ Departamento de Análises Clínicas e Toxicológicas - Faculdade de Farmácia - Universidade Federal de Minas Gerais,

Belo Horizonte-MG.

${ }^{2}$ Laboratório de Patologia Clínica - Hospital Governador Israel Pinheiro (Ipsemg) - Belo Horizonte-MG.

Correspondência: Maria das Graças Carvalho

Laboratório de Hematologia, Departamento de Análises Clínicas e Toxicológicas

Faculdade de Farmácia - Universidade Federal de Minas Gerais

Av. Antônio Carlos, 6627 - Pampulha

31270-010 - Belo Horizonte-MG - Brasil

Tel: (31) 3409-6881; Fax: (31) 3409-6895

E-mail:mgcarvalho@farmacia.ufmg.br 
nas crianças e diminuição da capacidade de trabalho em adultos. ${ }^{6-9}$ Finalmente, a anemia de doença crônica, considerada, em parte, como um defeito no metabolismo do ferro, ${ }^{10}$ apresenta três grandes causas clínicas: infecção, inflamação e neoplasia, que são responsáveis por $75 \%$ dos casos. ${ }^{11} \mathrm{~A}$ anemia de doença crônica é a anemia mais freqüente em pacientes hospitalizados. ${ }^{12,13}$

O diagnóstico diferencial das anemias microcíticas e hipocrômicas se reveste de grande importância, uma vez que cada uma delas apresenta causa, patogênese, prognóstico e tratamento inteiramente diferentes. Atualmente, o protocolo aceito para o diagnóstico das anemias citadas anteriormente envolve os chamados exames padrão-ouro, entre os quais aqueles avaliadores do metabolismo do ferro e a eletroforese de hemoglobina. Apesar de sua grande utilidade, estes testes são freqüentemente onerosos e demandam tempo. ${ }^{14} \mathrm{Com}$ o propósito de tornar mais simples e eficiente o diagnóstico diferencial, índices derivados dos modernos contadores automáticos têm sido sugeridos. Bessman et al. ${ }^{15}$ sugeriram que o RDW poderia contribuir para uma melhor diferenciação das anemias microcíticas e hipocrômicas. O RDW, um índice de anisocitose eritrocitária, representa a heterogeneidade de distribuição do tamanho das hemácias, diferenciando aquelas anemias com uma população homogênea de eritrócitos, entre elas a talassemia menor e anemia de doença crônica, daquelas que apresentam uma população heterogênea (anemia ferropriva). ${ }^{16} \mathrm{O}$ propósito deste estudo foi investigar a acurácia diagnóstica do RDW na diferenciação destes tipos de anemia.

\section{Casuística e Método}

Após consentimento livre e esclarecido, foram coletadas amostras de 159 pacientes atendidos no Serviço de Patologia Clínica do Hospital Governador Israel Pinheiro, com idade superior a 18 anos e hemoglobina inferior a $12,0 \mathrm{~g} / \mathrm{dL}$ para mulheres e 13,0 g/dL para homens. Os níveis séricos de ferro, a capacidade total de ligação do ferro à transferrina e a ferritina foram determinados, além da realização da eletroforese de hemoglobina. Os níveis de ferro sérico e capacidade de ligação do ferro foram determinados no aparelho Dimension $\mathrm{AR} \otimes$, da Dade Bhering, por métodos colorimétricos, usando-se kits da marca Dade Bhering com os seguintes valores de referência: ferro sérico (35 a $150 \mu \mathrm{g} / \mathrm{dL})$; capacidade de ligação do ferro $(250$ a $450 \mu \mathrm{g} / \mathrm{dL})$. A concentração de ferritina sérica foi medida no aparelho Immulite $\AA$ da DPC $₫$, que utiliza o método de quimioluminescência e kits Immulite 2000 da DPC $®$ (Diagnostic Products Corporation) cujos valores de referência são 28 a $397 \mathrm{ng} / \mathrm{mL}$ para homens e 6 a 159 ng/mL em mulheres. A eletroforese de hemoglobina foi realizada usando-se fitas de acetato de celulose, em pH alcalino, 8,6, em cuba Fonte Eletroforese Tecnow ${ }^{\circledR} 7000$. Em caso de suspeita de aumento de $\mathrm{HbA}_{2}$, foi realizada a sua dosagem pelo método de eluição da fita eletroforética. A avaliação hematológica foi realizada no contador automático Advia ${ }^{\circledR}$ 120 da Bayer ${ }^{\circledR}$. Com base nos resultados dos exames citados anteriormente, os pacientes foram classificados em três grupos a saber: anemia ferropriva, anemia de doença crônica e $\beta$ talassemia menor. Os pacientes com anemia microcítica e hipocrômica foram classificados como portadores de anemia ferropriva quando apresentaram níveis baixos de ferritina (inferiores a 6 e $28 \mathrm{ng} / \mathrm{mL}$ em mulheres e homens, respectivamente). Os pacientes com anemia microcítica e hipocrômica foram classificados, a princípio, como portadores de anemia da doença crônica quando apresentaram níveis de ferritina aumentados ou normais, capacidade total de ligação do ferro e ferro sérico normais ou diminuídos. Esses pacientes foram posteriormente avaliados quanto a seus dados clínicos e epidemiológicos quando disponíveis. Os pacientes com anemia microcítica e hipocrômica foram classificados como portadores de $\beta$ talassemia menor quando apresentaram níveis séricos de ferritina e ferro normais, porém dosagem de hemoglobina $\mathrm{A}_{2}$ aumentada (superior a 3,5 g/dL). Baseando-se nestes critérios, 83 pacientes puderam ser classificados como portadores de anemia ferropriva, 53 como portadores de anemia de doença crônica e 23 como portadores de $\beta$ talassemia menor.

Os dados referentes ao RDW foram submetidos a uma análise estatística usando-se o Programa Minitab 14. A análise de variância (ANOVA) foi usada para identificar as diferenças entre os três grupos. Um valor de $\mathrm{P}<0,01$ foi considerado estatisticamente significativo.

\section{Resultados}

O grupo de pacientes portadores de anemia ferropriva apresentou média de idade de 50 anos e se compôs de 66 pacientes do sexo feminino e 17 do sexo masculino. No grupo anemia de doença crônica, a média de idade foi de 58 anos e o grupo apresentava, em sua composição, 27 pacientes do sexo masculino e 26 do sexo feminino. Finalmente, no grupo dos portadores de $\beta$ talassemia menor, a média de idade foi de 60 anos, sendo que este grupo possuía 18 pacientes do sexo feminino e cinco do sexo masculino. A caracterização dos pacientes estudados é apresentada na Tabela 1 .

Em relação ao parâmetro RDW (valor de referência de $11,6 \%$ a $14,8 \%$ no contador Advia ${ }^{\circledR} 120$ da Bayer $\left.{ }^{\circledR}\right)$, os pacientes do grupo anemia ferropriva mostraram valores médios de $16,4 \pm 1,6 \%$, enquanto no grupo de pacientes portadores de anemia de doença crônica este valor foi de 16,0 $\pm 2,4 \%$. E, por último, os valores médios para o RDW nos pacientes $\operatorname{com} \beta$ talassemia menor foram de 15,6 $\pm 1,7 \%$. Embora tenha havido uma leve tendência de maior RDW na anemia ferropriva, não foram verificadas diferenças estatisticamente significativas entre os três grupos. As distribuições dos valores do RDW nos 83 pacientes com anemia ferropriva, 53 pacientes portadores de anemia de doença crônica e $23 \beta$ talassêmicos do tipo menor são mostradas na Figura 1. 
Tabela 1. Caracterização dos pacientes com relação à média de idade (idades mínima e máxima) e ao sexo

\begin{tabular}{ccccc}
\hline & \multirow{2}{*}{$\begin{array}{c}\text { Idade } \\
(\text { anos })\end{array}$} & $\begin{array}{c}\text { Masculino } \\
(\mathrm{n}=48)\end{array}$ & $\begin{array}{c}\text { Feminino } \\
(\mathrm{n}=111)\end{array}$ & $\begin{array}{c}\text { Total } \\
(\mathrm{n}=159)\end{array}$ \\
\cline { 3 - 4 } & & $17(20,5 \%)$ & $66(79,5 \%)$ & 83 \\
AF & $50(20-81)$ & 170 & 53 \\
ADC & $58(24-85)$ & $26(49,1 \%)$ & $27(50,9 \%)$ & 53 \\
\hline -Tal & $60(26-77)$ & $5(21,7 \%)$ & $18(78,3 \%)$ & 23 \\
\hline
\end{tabular}

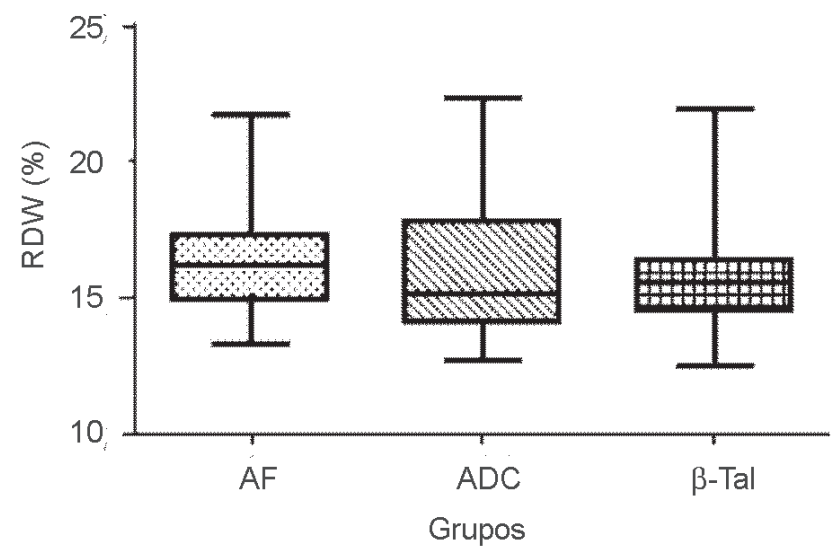

Figura 1. Distribuição dos valores de RDW em 83 pacientes com anemia ferropriva, 53 pacientes com anemia de doença crônica e 23 pacientes portadores de $\beta$ talassemia menor

\section{Discussão}

Na população envolvida neste estudo, a anemia ferropriva, $\beta$ talassemia menor e anemia de doença crônica figuram entre as anemias microcíticas e hipocrômicas mais comuns. Atualmente, o diagnóstico destas anemias se baseia nos chamados exames padrão-ouro que, apesar de úteis, são onerosos e demandam tempo. Uma limitação destes métodos consiste na interpretação dos resultados quando ocorre concomitância de doenças. Um índice simplificado que pudesse distinguir de maneira confiável as anemias microcíticas seria uma ferramenta extremamente útil. Bessman et al.$^{15}$ propuseram que o RDW, um parâmetro disponível nos modernos contadores automáticos, poderia melhorar a classificação dos pacientes portadores das referidas anemias com base na heterogeneidade de distribuição do tamanho do eritrócito. Neste caso, as anemias se enquadrariam em dois grupos, a saber: aquelas com uma população de hemácias heterogênea, entre elas a anemia ferropriva, e aquelas possuidoras de uma população homogênea de hemácias, citando-se, neste caso, as talassemias do tipo menor e a anemia de doença crônica. No caso da anemia de doença crônica, a presença de anisocitose e poiquilocitose são incomuns, e o RDW normal reflete a homogeneidade da população de células sangüíneas vermelhas. ${ }^{16}$
No caso das talassemias do tipo menor, como verificado por Bessman \& Feinstein, ${ }^{17}$ os eritrócitos são mais homogêneos quando comparados aos dos pacientes com anemia ferropriva. Conseqüentemente, o RDW tende a ser maior na anemia ferropriva do que nas talassemias menores. ${ }^{18} \mathrm{Na} \alpha \mathrm{e} \beta$ talassemias tipo menor, quase todos os eritrócitos são microcíticos devido a uma síntese deficiente de cadeias globínicas resultante de mutações que são expressas igualmente em todos os precursores de hemácias tornando os valores de RDW relativamente constantes. ${ }^{19} \mathrm{O}$ mesmo não ocorre na anemia ferropriva, onde a anisocitose verificada é devido à coexistência, no sangue periférico, de células sangüíneas vermelhas produzidas na medula óssea durante estágios progressivos de deficiência do ferro, dando lugar a uma população variada de eritrócitos, incluindo normocíticos e aqueles progressivamente microcíticos. ${ }^{20} \mathrm{O}$ aporte intermitente de ferro à medula óssea, reflexo da progressiva redução das reservas de ferro do organismo e da ingesta desigual de ferro, em dias sucessivos, vem corrobar também para o aumento da anisocitose.

Contudo, existem controvérsias sobre a real eficácia do RDW como parâmetro auxiliar na distinção das anemias. Yermiahu et al. ${ }^{21}$ e Aslan et al..$^{19}$ verificaram a existência de diferença estatisticamente significativa para o RDW entre os pacientes com anemia ferropriva e $\beta$ talassemia menor. O mesmo não foi encontrado nos trabalhos de Flynn et al., ${ }^{22}$ Tillyer \& Tillyer ${ }^{23}$ e Lafferty et al. ${ }^{24}$ que provaram ser o RDW um índice com pequeno poder discriminatório e contribuição limitada na diferenciação dos estados microcíticos. Eldibany et al. ${ }^{25}$ encontraram valores aumentados de RDW em ambas, talassemia menor (dos tipos $\alpha$ e $\beta$ ) e anemia ferropriva. No estudo de Demir et al ${ }^{26}$ foi observada uma pequena acurácia diagnóstica para o RDW, uma vez que este parâmetro classificou corretamente apenas 59\% dos pacientes. Em trabalho mais recente investigando a diferenciação entre talassemia menor e anemia ferropriva, conduzido por Alfadhli et al. ${ }^{14} \mathrm{O}$ RDW também não se mostrou bastante eficiente dentre as nove funções avaliadas. No Brasil, Lima et al.,${ }^{27}$ avaliando a utilidade clínica do RDW, verificaram que $90 \%$ dos pacientes com anemia ferropriva e $77 \%$ dos pacientes $\operatorname{com} \beta$ talassemia menor foram corretamente identificados usando valores de RDW superiores ou inferiores a $21 \%$, respectivamente. Sob a ótica de tais resultados concluiu-se que o RDW poderia ser um método específico e sensível para identificar a anemia ferropriva entre pacientes apresentando um quadro de microcitose; contudo, este parâmetro não seria um bom índice para a identificação da $\beta$ talassemia menor. Desta forma, uma distinção clara entre estes distúrbios não poderia ser feita baseando-se apenas no RDW.

Neste estudo não foi verificada diferença significativa para o RDW entre os três grupos de anemias microcíticas avaliadas, indicando ter este índice uma limitada utilidade para a diferenciação entre anemia ferropriva, $\beta$ talassemia menor e anemia de doença crônica, sinalizando para a neces- 
sidade de se envidar esforços no sentido do desenvolvimento de outro índice mais eficiente. Enquanto isto não ocorre, a utilização dos métodos padrão ouro no diagnóstico diferencial continua imprescindível na prática médica.

\begin{abstract}
Iron deficiency anemia, the thalassemia trait and chronic disease anemia are the most common microcytic and hypochromic anemias in the Brazilian population. Differential diagnosis of these anemias is of great clinical importance however, frequently, it is complex due to coexistence of diseases, as well as being time consuming and expensive. In order to simplify and increase efficiently of checking the differential diagnoses of these anemias, the use of indexes derived from modern blood cell counters has been suggested. Among them, is the index called red blood cell distribution width which indicates the variability in red blood cell size. In this study, the discriminative power of the red blood cell distribution width in differentiating microcytic and hypochromic anemias was evaluated in a group of 159 patients diagnosed as carriers of either iron deficiency anemia, $\beta$-thalassemia minor or chronic disease anemia. No difference was found for the red blood cell distribution width among the three groups of microcytic and hypochromic anemias indicating that this index is not a useful tool to distinguish among iron deficiency, $\beta$ thalassemia minor and chronic disease anemia. Rev. bras. hematol. hemoter. 2008; 30(2):120-123.
\end{abstract}

Key words: Iron deficiency anemia; chronic disease anemia; $\beta$ thalassemia minor

\section{Agradecimentos}

Nossos sinceros agradecimentos à equipe do Laboratório de Patologia Clínica do Hospital Governador Israel Pinheiro (Ipsemg) pela seleção das amostras de sangue dos pacientes.

\section{Referências Bibliográficas}

1. Zago MA, Falcão RP, Pasquini R. Hematologia: Fundamentos e prática. Ed. Rev. Atual. São Paulo: Atheneu, 2005. 1081p.

2. Tuzmen S, Schechter AN. Genetic diseases of hemoglobin: diagnostic methods for elucidating beta-thalassemia mutations. Blood Rev. 2001;15(1):19-29.

3. Sarnaik SA. Thalassemia and related hemoglobinopathies. Indian J Pediatr. 2005;72(4):319-24.

4. Coutinho GG, Goloni-Bertollo EM, Bertelli EC. Sarnaik SA. Iron deficiency anemia in children: a challenge for public health and for society. São Paulo Med J. 2005;123(2):88-92.

5. World Health Organization - WHO. Disponível em: $<$ http://www. who.int/water_sanitation_health/diseases/anemia en>. Acesso em: 10 fev.2007.

6. Nobili B, Perrotta S, Matarese SMR, Conte ML, Giudice EM. Evaluation of body iron status in Italian carriers of beta-thalassemia trait. Nutr Res. 2001;21:55-60.

7. Horton S, Ross J. The economics of iron deficiency. Food Policy. 2003;28:51-75.

8. Oppenheimer SJ. Iron and its relation to immunity and infectious disease. 2001;131(2S-2):616S-633S.

9. Lorenzi TF. Manual de Hematologia - Propedêutica e Clínica. $3^{\mathrm{a}}$ ed. Rio de Janeiro: Medsi, 2003. 655p.
10. Means RT Jr, Krantz SB. Progress in understanding the pathogenesis of the anemia of chronic disease. Blood. 1992;80(7):1639-47.

11. Fitzsimons EJ, Brock JH. The anaemia of chronic disease. BMJ 2001;322(7290):811-12.

12. Weiss G. Pathogenesis and treatment of anaemia of chronic disease. Blood Rev. 2002;16(2):87-96.

13. Jayaranee S, Sthaneshwar P. Serum soluble transferrin receptor in hypochromic microcytic anaemia. Singapore Med J. 2006;47(2): 138-42.

14. AlFadhli SM, Al-Awadhi AM, AlKhaldi D. Validity assessment of nine discriminant functions used for the differentiation between iron deficiency anemia and thalassemia minor. J Trop Pediatr. 2007;53(2):93-7

15. Bessman JD, Gilmer PR Jr., Gardner FH. Improved classification of anemias by MCV and RDW. Am J Clin Pathol. 1983;80(3):322-326.

16. Karnad A, Poskitt TR. The automated complete blood cell count. Use of the red blood cell volume distribution width and mean platelet volume in evaluating anemia and thrombocytopenia. Arch Intern Med. 1985;145(7):1270-2.

17. Bessman JD, Feinstein DI. Quantitative anisocytosis as a discriminant between iron deficiency and thalassemia minor. Blood 1979; 53(2):288-93.

18. Trent RJ. Diagnosis of the haemoglobinopathies. Clin Biochem Rev. 2006;27(1):27-38.

19. Aslan D, Gümrük F, Gürgey A, Altay C. Importance of RDW value in differential diagnosis of hypochrome anemias. Am J Hematol 2002;69(1):31-3.

20. Green R, King R. A new red cell discriminant incorporating volume dispersion for differentiating iron deficiency anemia from thalassemia minor. Blood Cells. 1989;15(3):481-95.

21. Yermiahu T, Ben-Shalom M, Porath A, Vardi H, Boantza A, Mazor $\mathrm{D}$, et al. Quantitative determinations of microcytic-hypochromic red blood cell population and glycerol permeability in iron deficiency anemia and beta thalassemia minor. Ann Hematol 1999; 78(10):468-71.

22. Flynn MM, Reppun TS, Bhagavan NV. Limitations of red blood cell distribution width (RDW) in evaluation of microcytosis. Am J Clin Pathol. 1986;85(4):445-9.

23. Tillyer ML, Tillyer CR. Use of red cell distribution width and erythrocyte zinc protoporphyrin in differential diagnosis of $\alpha$ and $\beta$ thalassaemia and iron deficiency. J Clin Pathol 1994;47(3): 205-8.

24. Lafferty JD, Crowther MA, Ali MA, Levine M. The evaluation of various mathematical $\mathrm{RBC}$ indices and their efficacy in discriminating between thalassemic and non-thalassemic microcytosis. Am J Clin Pathol. 1996;106(2):201-5.

25. Eldibany MM, Totonchi KF, Joseph NJ, Rhone D. Usefulness of certain red blood cell indices in diagnosing and differentiating thalassemia trait from iron-deficiency anemia. Am J Clin Pathol. 1999;111(5):676-82.

26. Demir A, Yarali N, Fisgin T, Duru F, Kara A. Most reliable indices in differentiation between thalassemia trait and iron deficiency anemia. Pediatr Int. 2002;44(6):612-6.

27. Lima CS, Reis AR, Grotto HZ, Saad ST, Costa FF. Comparison of red cell distribution width and a red cell discriminant function incorporating volume dispersion for distinguishing iron deficiency from beta thalassemia trait in patients with microcytosis. São Paulo Med J. 1996;114(5):1265-9.

Avaliação: Editor e dois revisores externos

Conflito de interesse: não declarado

Recebido: 05/03/2007

Aceito: 18/09/2007 